\title{
Effect of high and low roughage total mixed ration diets on rumen metabolites and enzymatic profiles in crossbred cattle and buffaloes
}

\author{
S. K. Sinha ${ }^{1,2}$, V. B. Chaturvedi ${ }^{1}$, Putan Singh ${ }^{1}$, L. C. Chaudhary ${ }^{1}$, Mayukh Ghosh ${ }^{3}$ and Swati Shivani ${ }^{4}$
}

1. Division of Animal Nutrition, ICAR-Indian Veterinary Research Institute, Izatnagar, Bareilly - 243122 , Uttar Pradesh, India; 2. Department of Animal Nutrition, Ranchi Veterinary College, Birsa Agricultural University, Kanke,

Ranchi - 834 006, Jharkhand, India; 3. Department of Veterinary Biochemistry, Ranchi Veterinary College, Birsa Agricultural University, Kanke, Ranchi - 834 006, Jharkhand, India; 4. Division of Animal Nutrition, ICAR-National Dairy Research Institute, Karnal - 132 001, Haryana, India.

Corresponding author: S. K. Sinha, e-mail: subodh.rvc@gmail.com

Co-authors: VBC: chaturvedivb@gmail.com, PS: putan60@gmail.com, LCC: Icchaudhary1@rediffmail.com, MG: ghosh.mayukh87@gmail.com, SS: swatishivani2703@gmail.com

Received: 26-11-2016, Accepted: 19-04-2017, Published online: 09-06-2017

doi: 10.14202/vetworld.2017.616-622 How to cite this article: Sinha SK, Chaturvedi VB, Singh P, Chaudhary LC, Ghosh M, Shivani S (2017) Effect of high and low roughage total mixed ration diets on rumen metabolites and enzymatic profiles in crossbred cattle and buffaloes, Veterinary World, 10(6):616-622.

\begin{abstract}
Aim: A comparative study was conducted on crossbred cattle and buffaloes to investigate the effect of feeding high and low roughage total mixed ration (TMR) diets on rumen metabolites and enzymatic profiles.

Materials and Methods: Three rumen-fistulated crossbred cattle and buffalo were randomly assigned as per $3 \times 3$ switch over design for 21-days. Three TMR diets consisting of concentrate mixture, wheat straw and green maize fodder in the ratios of $\left(\mathrm{T}_{1}\right)$ 60:20:20, $\left(\mathrm{T}_{2}\right)$ 40:30:30, and $\left(\mathrm{T}_{3}\right)$ 20:40:40, respectively, were fed to the animals ad libitum. Rumen liquor samples were collected at $0,2,4,6$, and $8 \mathrm{~h}$ post feeding for the estimation of rumen biochemical parameters on 2 consecutive days in each trial.

Results: The lactic acid concentration and $\mathrm{pH}$ value were comparable in both species and treatments. Feed intake $(99.77 \pm 2.51 \mathrm{~g} / \mathrm{kg}$ body weight), ruminal ammonia nitrogen, and total nitrogen were significantly $(\mathrm{p}<0.05)$ higher in buffalo and in treatment group fed with high concentrate diet. Production of total volatile fatty acids (VFAs) was non-significant $(\mathrm{p}>0.05)$ among treatments and significantly $(\mathrm{p}<0.05)$ greater in crossbred cattle than buffaloes. Molar proportions of individual VFAs propionate $(\mathrm{C} 3)$, propionate:butyrate $(\mathrm{C} 3: \mathrm{C} 4)$, and (acetate+butyrate):propionate $([\mathrm{C} 2+\mathrm{C} 4]$ : $\mathrm{C} 3)$ ratio in both crossbred cattle and buffalo were not affected by high or low roughage diet, but percentage of acetate and butyrate varied significantly $(\mathrm{p}<0.05)$ among treatment groups. Activities of microbial enzymes were comparable among species and different treatment groups. A total number of rumen protozoa were significantly $(\mathrm{p}<0.05)$ higher in crossbred cattle than buffaloes along with significantly $(\mathrm{p}<0.05)$ higher population in animal fed with high concentrate diet $\left(\mathrm{T}_{1}\right)$.

Conclusion: Rumen microbial population and fermentation depend on constituents of the treatment diet. However, microbial enzyme activity remains similar among species and different treatments. High concentrate diet increases number of rumen protozoa, and the number is higher in crossbred cattle than buffaloes.
\end{abstract}

Keywords: buffalo, crossbred cattle, rumen ecology, rumen metabolites.

\section{Introduction}

The comparison of rumen metabolism of crossbred cattle and buffaloes are quite challenging and interesting to recognize the rumen microbial activities under the same feeding and environmental conditions. The rumen in both cattle and buffaloes is well developed and recognized as fermentation vat to utilize the cellulosic matter and allows the maximal use of fermentation end-products particularly volatile fatty acids (VFAs) and microbial proteins for ruminants [1-4]. Despite these similarities, some differences also exists in terms of feed intake, nutrient digestibility, rumen

Copyright: Sinha, et al. Open Access. This article is distributed under the terms of the Creative Commons Attribution 4.0 International License (http://creativecommons.org/licenses/by/4.0/), which permits unrestricted use, distribution, and reproduction in any medium, provided you give appropriate credit to the original author(s) and the source, provide a link to the Creative Commons license, and indicate if changes were made. The Creative Commons Public Domain Dedication waiver (http://creativecommons.org/ publicdomain/zero/1.0/) applies to the data made available in this article, unless otherwise stated. bacterial and protozoal population, behavioral habits, and their interactions with the environment $[1,5,6]$.

Many in-vitro as well as in-vivo studies have been carried out concerning digestion, metabolism, rumen microbial population, and physiological conditions in cattle and buffaloes [7-14]. Swamp buffaloes are more efficient than cattle in many aspects, namely, nitrogen (N)-recycling and fiber digestion, ruminal ammonia nitrogen $\left(\mathrm{NH}_{3}-\mathrm{N}\right)$ level in relation to efficient fermentation and intake [1,15-17]. High grain diet and or the little addition of soluble carbohydrate results in shift of fermentation pattern, lowered ruminal $\mathrm{pH}$, and few protozoa may be eliminated or inhibited [18].

There is paucity of literature dealing with rearing of cattle and buffalo on similar diets and under same environmental conditions. Therefore, the present study was designed to evaluate the effect of feeding high and low roughage total mixed ration diets on rumen metabolites and enzymatic profiles in crossbred cattle and buffaloes. 


\section{Materials and Methods}

\section{Ethical approval}

All the animal experiments were conducted after approval of Institute Animal Ethics Committee of Indian Veterinary Research Institute (IVRI) after approval by the Director IVRI and CPCSEA, Ministry of Environment and Forestry, Government of India.

\section{Animals, diets, and experimental design}

Three crossbred cattle (Bos taurus $\times$ Bos indicus) and buffaloes (Bubalus bubalis) having average body weight of $364 \pm 12$ and $335 \pm 8 \mathrm{~kg}$, respectively, with permanent fistula were selected. Animals were randomly assigned to receive three treatments of 21 days as per $3 \times 3$ switch-over design. The concentrate with roughage was fed ad libitum in different ratios according to their body weight. The dietary treatments (concentrate mixture, wheat straw, and green fodder) simulated three different feeding systems, viz., $\left(\mathrm{T}_{1}\right)$ 60:20:20, $\left(\mathrm{T}_{2}\right)$ 40:30:30, and $\left(\mathrm{T}_{3}\right)$ 20:40:40 were applied (Table-1). Concentrate mixture consisted crushed maize grain (37\%), solvent-extracted soybean meal $(20 \%)$, wheat bran $(40 \%)$, mineral mixture $(2 \%)$, and salt (1\%) and analyzed composition of concentrate and roughage have been presented in Table-2.

\section{Sampling and analysis of rumen fluid}

During the past two consecutive days of the experiment, rumen fluids $(100 \mathrm{ml})$ were collected into a pre-warmed flask from the fistulated animals at 0 , 2, 4, 6 and $8 \mathrm{~h}$ post-feeding of each animal. Ruminal digestibility and rumen metabolite concentrations are changed after feeding in a time-dependent manner. Hence, comparison of effect of high and low roughage diet on rumen metabolite and enzymatic profile will also change in due to the course of time post-feeding. Thus, we have considered different time frame to observe the effect of diet based on the mean values of different rumen metabolite and enzymatic parameters. Moreover, digestibility depends on so many factors including interspecies variation. Monitoring different rumen metabolite parameters and enzymatic profile in response to different types of diet, the interspecies variation can be assumed form which the diet and ruminal environment can be manipulated to have a better effect on production status from these animals. Hence, we thought species interaction might add good values to the current research. Immediately, after collection $\mathrm{pH}$ of rumen fluid was measured and transported to laboratory for further analysis such as protozoa, VFAs, and other biochemical parameters related to $\mathrm{N}$ fractions. Some amounts of content were kept at $-20^{\circ} \mathrm{C}$ for enzymatic study. $\mathrm{NH}_{3}-\mathrm{N}$ was estimated by method described earlier by Weatherburn [19].

\section{Rumen metabolites}

Rumen fluid was separated into two parts; one part was used to determine VFAs which were analyzed using gas chromatograph equipped with a double flame ionization detector [20] and the second part was analyzed for $\mathrm{NH}_{3} \mathrm{~N}$, total $\mathrm{N}(\mathrm{TN})$, and trichloroacetic
Table-1: Analysed composition (\%) of concentrate and roughage.

\begin{tabular}{lccc}
\hline Attributes & $\begin{array}{c}\text { Concentrate } \\
\text { mixture (C) }\end{array}$ & $\begin{array}{c}\text { Wheat } \\
\text { straw (W) }\end{array}$ & $\begin{array}{c}\text { Green } \\
\text { maize (G) }\end{array}$ \\
\hline Proximate & & & \\
components & & & \\
DM & 89.13 & 91.57 & 18.97 \\
OM & 92.51 & 92.65 & 91.21 \\
CP & 20.13 & 2.95 & 9.22 \\
EE & 2.80 & 0.84 & 1.01 \\
Cell wall & & & \\
components & & & \\
CF & 8.35 & 34.47 & 38.64 \\
NDF & 37.36 & 74.52 & 62.97 \\
ADF & 10.35 & 52.84 & 39.61 \\
\hline DM
\end{tabular}

$\mathrm{DM}=$ Dry matter; $\mathrm{OM}=$ Organic matter; $\mathrm{CP}=$ Crude protein; $\mathrm{EE}=$ Ether extract; $\mathrm{CF}=$ Crude fiber; $\mathrm{NDF}=$ Neutral detergent fiber; $A D F=A c i d$ detergent fiber.

Table-2: Ingredient and chemical composition of diets (\% on dry matter basis).

\begin{tabular}{|c|c|c|c|}
\hline \multirow[t]{2}{*}{ Component } & TMR-I & TMR-II & TMR-III \\
\hline & $\begin{array}{l}\text { 60C:20W: } \\
20 G\end{array}$ & $\begin{array}{l}\text { 40C:30W: } \\
30 \mathrm{G}\end{array}$ & $\begin{array}{l}\text { 20C: } 40 W \\
40 G\end{array}$ \\
\hline \multicolumn{4}{|l|}{ Diet ingredients (\%) } \\
\hline $\begin{array}{l}\text { Concentrate } \\
\text { mixture }(\mathrm{C})\end{array}$ & 60 & 40 & 20 \\
\hline Wheat straw (W) & 20 & 30 & 40 \\
\hline Green maize (G) & 20 & 30 & 40 \\
\hline \multicolumn{4}{|l|}{$\begin{array}{l}\text { Chemical } \\
\text { composition }\end{array}$} \\
\hline Organic matter & 92.30 & 92.16 & 92.05 \\
\hline Crude protein & 14.64 & 11.65 & 8.89 \\
\hline Ether extract & 2.07 & 1.67 & 1.30 \\
\hline $\begin{array}{l}\text { Neutral detergent } \\
\text { fiber }\end{array}$ & 49.59 & 56.31 & 62.48 \\
\hline Acid detergent fiber & 24.34 & 32.01 & 39.06 \\
\hline Total ash & 7.69 & 7.83 & 7.95 \\
\hline
\end{tabular}

Mineral mixture 2 and common salt $1 \%$ as supplement; concentrate mixture $=$ crushed maize, 37\%; solvent extracted soybean meal, 20\%; wheat bran, $40 \%$; mineral mixture, $2 \%$ and salt, $1 \%$. TMR $=$ Total mixed ration

acid precipitable nitrogen (TCA ppt. N) by the standard Kjeldahl procedure [21]. The nonprotein nitrogen (NPN) was calculated as the difference between TN and TCA ppt. N. Lactic acid (LA) concentration in rumen liquor was estimated as per method described by Barker and Summerson [22].

\section{Enzyme assay}

The microbial enzymes from the rumen contents were extracted as per the method described by Hristov et al. [23]. For estimation of carboxymethylcellulase (CMCase) and xylanase activity, the reaction mixture $(1 \mathrm{ml}$ phosphate buffer [0.1 M, pH 6.8], $0.5 \mathrm{ml}$ enzyme, and $0.5 \mathrm{ml}$ of either CMCase [1.0\%] or xylan $[0.25 \%])$, were incubated at $39^{\circ} \mathrm{C}$ for 60 and $30 \mathrm{~min}$, respectively, and the amount of reducing sugars released were estimated [24]. The Avicelase activity was estimated by measuring the amount of reducing sugar (Avicel 1\%) released from Avicel using the reaction mixture ( $1 \mathrm{ml}$ phosphate buffer [0.1 M, pH 6.8], 
$1 \mathrm{ml}$ enzyme and $0.5 \mathrm{ml}$ of Avicel [1\%]) incubated at $39^{\circ} \mathrm{C}$ for $60 \mathrm{~min}$. Protein and protease estimation was carried out as per the method described by Lowry et al. [25], and protease activity was measured using azocasein as substrate [26].

\section{Ciliate protozoa}

The number of protozoa was counted as per the procedure described by Kamra et al. [27]. Number of protozoa/ml rumen liquor $\mathrm{N}=(\mathrm{n} \times \mathrm{A} \times \mathrm{D}) /(\mathrm{a} \times \mathrm{v})$.

\section{Statistical analysis}

The means of all parameters measured were statistically analyzed by analysis of variance procedure and means were statistically compared in rumen fluid at $0,2,4,6$, and $8 \mathrm{~h}$ post-feeding among different treatment groups (T1, T2 and T3), between both animal species (cattle and buffalo) and among treatment and species $(\mathrm{T} \times \mathrm{S})$ using the statistical software SPSS (version 20.0). Differences among treatments were analyzed by Duncan's multiple range test using the generalized linear model of Snedecor and Cochran [28].

\section{Results}

There was non-significant ( $p>0.05)$ difference in intake $\mathrm{g} / \mathrm{kgW}^{0.75}$ and digestibility of dry matter (DM), organic matter $(\mathrm{OM})$, crude protein $(\mathrm{CP})$, ether extract, neutral detergent fiber (NDF), and acid detergent fiber (ADF) in crossbred cattle and buffalo (Table-3). Among treatment, digestibility was influenced in high roughage diet to low roughage diet. In ruminant species, apparent digestibility was increased according to inclusion level of concentrate in diets.

\section{Rumen pH}

Feeding of different diet did not alter $\mathrm{pH}$ of the rumen fluid significantly $(\mathrm{p}>0.05)$ post-feeding among different treatment groups. No significant $(\mathrm{p}>0.05)$ variation in ruminal $\mathrm{pH}$ was also observed post-treatment between crossbred cattle and buffalo population (Table-4).

\section{Nitrogen fractions}

Nitrogen fractions, viz., $\mathrm{NH}_{3}-\mathrm{N}, \mathrm{TN}, \mathrm{TCA}$ ppt. $\mathrm{N}$ and NPN in the rumen liquor in groups $\left(\mathrm{T}_{1}, \mathrm{~T}_{2}\right.$, and $\mathrm{T}_{3}$ ) are presented in Table- 4 . The concentration of $\mathrm{NH}_{3}-\mathrm{N}(\mathrm{mg} / \mathrm{dL})$ was significantly $(\mathrm{p}<0.01)$ lower in group $\mathrm{T}_{3}$ followed by $\mathrm{T}_{2}$ than $\mathrm{T}_{1}$. Ruminal $\mathrm{NH}_{3}-\mathrm{N}$ varied significantly $(p<0.05)$ and was found to be higher in buffalo than crossbred cattle. The total nitrogen level $(\mathrm{mg} / \mathrm{dL})$ was found to be significantly $(\mathrm{p}<0.01)$ higher in buffaloes than crossbred cattle. Among treatments, it was varied significantly $(\mathrm{p}<0.01)$ and observed to be highest for $\mathrm{T}_{1}$ and lowest for $\mathrm{T}_{3}$; however, the value in $T_{2}$ was intermediate between these two groups. The variation in mean values of TCA ppt. N were observed to be non-significant ( $>0.05$ ) between crossbred cattle and buffaloes but it varied significantly $(\mathrm{p}<0.05)$ within the treatment groups. The NPN value $(\mathrm{mg} / \mathrm{dl})$ was significantly $(p<0.01)$ higher for buffaloes than crossbred cattle. Among the treatments groups, NPN value $(\mathrm{mg} / \mathrm{dL})$ was non-significant $(\mathrm{p}>0.05)$ with each other.

\section{LA and VFAs}

There was no significant $(p>0.05)$ variation observed in LA concentration, neither among the treatment groups nor between crossbred cattle and buffalo population post treatment (Table-4).

The values of total VFAs (TVFAs) and relative percent of acetate, propionate, and butyrate were depicted in Table-5. A significant difference $(\mathrm{p}<0.05)$ in TVFAs concentration was observed in the rumen liquor of crossbred cattle and buffaloes. The level of acetate was significantly $(\mathrm{p}<0.05)$ higher in crossbred cattle $(9.26 \pm 0.24)$ than buffaloes $(8.41 \pm 0.24)$. However, no significant ( $>0.05)$ variation in acetate concentration was observed among the treatment groups post-feeding. Whereas propionate concentrations did not differ significantly $(\mathrm{p}>0.05)$ among the treatment groups and between cross-bred cattle and buffalo population post-feeding. Butyrate ( $\mathrm{mmol} / \mathrm{dL})$

Table-3: Feed intakes and apparent digestibility (\%) of TMR in cattle and swamp buffaloes receiving the same diets.

\begin{tabular}{|c|c|c|c|c|c|c|c|c|c|}
\hline \multirow[t]{2}{*}{ Attributes } & \multirow{2}{*}{$\begin{array}{c}\text { 60C: } 20 \mathrm{~W}: \\
\text { 20G } \\
\text { T1 }\end{array}$} & \multirow{2}{*}{$\begin{array}{c}\text { 40C:30W: } \\
\text { 30G } \\
\text { T2 }\end{array}$} & \multirow{2}{*}{$\begin{array}{c}\begin{array}{c}20 \mathrm{C}: 40 \mathrm{~W}: \\
40 \mathrm{G}\end{array} \\
\text { T3 }\end{array}$} & \multicolumn{2}{|c|}{ Mean \pm SE } & \multirow[t]{2}{*}{ SEM } & \multicolumn{3}{|c|}{ p values } \\
\hline & & & & Cattle & Buffalo & & $\mathbf{T}$ & $\mathbf{S}$ & $\mathbf{T} \times \mathbf{S}$ \\
\hline DMI, kg/day & $6.93^{\mathrm{b}} \pm 0.34$ & $6.71^{\mathrm{ab}} \pm 0.34$ & $5.83^{\mathrm{a}} \pm 0.32$ & $5.91^{q} \pm 0.27$ & $7.06^{p} \pm 0.25$ & 0.20 & $0.04 *$ & $0.008 * *$ & 0.91 \\
\hline DMI g/kgW ${ }^{0.75}$ & $105.49^{b} \pm 1.76$ & $101.52^{b} \pm 2.77$ & $86.33^{a} \pm 2.35$ & $95.79 \pm 2.88$ & $99.77 \pm 2.51$ & 1.91 & $0.003 * *$ & 0.14 & 0.34 \\
\hline \multicolumn{10}{|l|}{$\begin{array}{l}\text { Apparent } \\
\text { digestibility, \% }\end{array}$} \\
\hline DM & $66.61^{c} \pm 0.57$ & $62.86^{b} \pm 0.82$ & $58.98^{a} \pm 0.97$ & $62.96 \pm 0.99$ & $62.68 \pm 0.99$ & 0.69 & $0.006 * *$ & 0.77 & 0.91 \\
\hline OM & $69.90^{\wedge} \pm 0.50$ & $67.48^{b} \pm 0.68$ & $65.32^{\mathrm{a}} \pm 0.77$ & $67.58 \pm 0.71$ & $67.55 \pm 0.69$ & 0.49 & $0.008 * *$ & 0.96 & 0.83 \\
\hline $\mathrm{CP}$ & $70.55^{b} \pm 1.52$ & $64.61^{\mathrm{ab}} \pm 2.35$ & $62.27^{a} \pm 2.51$ & $64.17 \pm 2.04$ & $67.45 \pm 1.74$ & 1.35 & $0.03 *$ & 0.20 & 0.73 \\
\hline $\mathrm{EE}$ & $72.42^{b} \pm 1.11$ & $63.49^{a} \pm 1.02$ & $62.76^{\mathrm{a}} \pm 2.04$ & $66.00 \pm 1.59$ & $66.45 \pm 1.58$ & 1.10 & $0.004 * *$ & 0.79 & 0.71 \\
\hline NDF & $49.16^{a} \pm 0.68$ & $52.16^{a} \pm 1.13$ & $56.68^{\mathrm{b}} \pm 2.07$ & $51.64 \pm 1.39$ & $53.69 \pm 1.28$ & 0.95 & $0.007 * *$ & 0.22 & 0.79 \\
\hline ADF & $41.06^{\mathrm{a}} \pm 1.47$ & $44.68^{\mathrm{ab}} \pm 1.54$ & $47.67^{b} \pm 1.43$ & $43.64 \pm 1.34$ & $45.30 \pm 1.34$ & 0.94 & $0.01 * *$ & 0.35 & 0.94 \\
\hline
\end{tabular}

Mean bearing different superscripts in a column and row differ significantly, $* p<0.05 ; * * p<0.01$. SEM=Standard error of the mean $(n=36), T=$ Dietary treatment, $S=$ Species (crossbred cattle and buffalo), $T \times S=$ Interaction between species and dietary treatments, $\mathrm{DMI}=$ Dry matter intake, $\mathrm{TMR}=$ Total mixed ration, $\mathrm{DM}=$ Dry matter, $\mathrm{OM}=$ Organic matter, $C P=$ Crude protein, $E E=$ Ether extract, $C F=$ Crude fiber, NDF=Neutral detergent fiber, ADF=Acid detergent fiber, $\mathrm{SE}=$ Standard error, $\mathrm{C}=$ Concentrate mixture, $\mathrm{W}=$ Wheat straw, $\mathrm{G}=\mathrm{Green}$ maize 
Table-4: Nitrogen fractions and lactate $(\mathrm{mg} / \mathrm{dL})$ in rumen liquor of fistulated crossbred cattle and buffaloes fed various TMR diet.

\begin{tabular}{|c|c|c|c|c|c|c|c|c|c|}
\hline \multirow[t]{2}{*}{ Attributes } & \multirow{2}{*}{$\begin{array}{c}\text { 60C:20W: } \\
20 \mathrm{G} \\
\mathrm{T1}\end{array}$} & \multirow{2}{*}{$\begin{array}{c}\text { 40C:30W: } \\
\text { 30G } \\
T 2\end{array}$} & \multirow{2}{*}{$\begin{array}{c}\text { 20C:40W: } \\
\text { 40G } \\
\text { T3 }\end{array}$} & \multicolumn{2}{|c|}{ Mean \pm SE } & \multirow[t]{2}{*}{ SEM } & \multicolumn{3}{|c|}{ p values } \\
\hline & & & & Cattle & Buffalo & & $\mathbf{T}$ & $\mathbf{S}$ & $\mathbf{T} \times \mathbf{S}$ \\
\hline $\mathrm{pH}$ & $6.24 \pm 0.05$ & $6.26 \pm 0.05$ & $6.33 \pm 0.07$ & $6.24 \pm 0.05$ & $6.31 \pm 0.04$ & 0.03 & 0.587 & 0.342 & 0.795 \\
\hline $\mathrm{NH}_{3}-\mathrm{N}$ & $11.11^{\mathrm{b}} \pm 0.66$ & $8.43^{a} \pm 0.42$ & $7.36^{a} \pm 0.44$ & $8.27^{a} \pm 0.53$ & $9.66^{p} \pm 0.55$ & 0.39 & $0.009 * *$ & $0.023 *$ & 0.909 \\
\hline $\mathrm{TN}^{3}$ & $82.83^{c} \pm 2.32$ & $65.83^{b} \pm 3.81$ & $58.00^{\mathrm{a}} \pm 3.24$ & $62.00^{\circ} \pm 3.51$ & $75.77^{p} \pm 2.80$ & 2.50 & $0.006 * *$ & $0.003 * *$ & 0.257 \\
\hline TCA-ppt. N & $58.50^{\mathrm{b}} \pm 2.54$ & $45.00^{\mathrm{a}} \pm 2.35$ & $40.33^{a} \pm 2.58$ & $47.33 \pm 2.76$ & $48.55 \pm 2.71$ & 1.91 & $0.007 * *$ & 0.684 & 0.959 \\
\hline NPN & $24.33 \pm 3.28$ & $20.83 \pm 4.00$ & $17.66 \pm 2.80$ & $14.66^{a} \pm 1.72$ & $27.22^{p} \pm 2.86$ & 1.96 & 0.29 & $0.005^{* *}$ & 0.406 \\
\hline LA & $1.76 \pm 0.04$ & $1.71 \pm 0.04$ & $1.66 \pm 0.07$ & $1.74 \pm 0.05$ & $1.68 \pm 0.04$ & 0.03 & 0.463 & 0.396 & 0.893 \\
\hline
\end{tabular}

Mean bearing different superscripts in a column and row differ significantly, $* \mathrm{p}<0.05 ; * * \mathrm{p}<0.01 ; \mathrm{mg} / \mathrm{dL}=\mathrm{Milligrams}$ per decilitre, $\mathrm{pH}=$ Potential of hydrogen, $\mathrm{NH} 3-\mathrm{N}=$ Ammonia nitrogen, $\mathrm{TN}=$ Total nitrogen, $\mathrm{TCA}-\mathrm{ppt}$. $\mathrm{N}=$ Trichloroacetic acid induced nitrogen precipitation, NPN=Non protein nitrogen, $\mathrm{LA}=$ Lactic acid, TMR=Total mixed ration, $\mathrm{SEM}=\mathrm{Standard}$ error of mean, $\mathrm{SE}=\mathrm{Standard}$ error, $\mathrm{T}=$ Dietary treatment, $\mathrm{S}=\mathrm{Species}$ (crossbred cattle and buffalo), $\mathrm{T} \times \mathrm{S}=\mathrm{Interaction}$ between species and dietary treatments, $\mathrm{C}=$ Concentrate mixture, $\mathrm{W}=$ Wheat straw, $\mathrm{G}=$ Green maize

Table-5: Effect on TVFA and its fractions $(\mathrm{mM} / 100 \mathrm{ml})$ and molar proportion $(\mathrm{mol} / 100 \mathrm{~mol})$ in rumen liquor of fistulated crossbred cattle and buffaloes fed various TMR diet.

\begin{tabular}{|c|c|c|c|c|c|c|c|c|c|}
\hline \multirow[t]{2}{*}{ Attributes } & \multirow{2}{*}{$\begin{array}{c}\text { 60C:20W: } \\
\text { 20G } \\
\text { T1 }\end{array}$} & \multirow{2}{*}{$\begin{array}{c}\text { 40C:30W: } \\
\text { 30G } \\
\text { T2 }\end{array}$} & \multirow{2}{*}{$\begin{array}{c}\text { 20C:40W: } \\
\text { 40G } \\
\text { T3 }\end{array}$} & \multicolumn{2}{|c|}{ Mean \pm SE } & \multirow[t]{2}{*}{ SEM } & \multicolumn{3}{|c|}{ p values } \\
\hline & & & & Cattle & Buffalo & & $\mathbf{T}$ & $\mathbf{s}$ & $\mathbf{T} \times \mathbf{S}$ \\
\hline Acetate, C2 & $8.95 \pm 0.26$ & $9.02 \pm 0.39$ & $8.54 \pm 0.28$ & $9.26^{p} \pm 0.24$ & $8.41^{a} \pm 0.24$ & 0.18 & 0.47 & $0.02 *$ & 0.32 \\
\hline Propionate, C3 & $2.16 \pm 0.12$ & $2.13 \pm 0.15$ & $2.00 \pm 0.12$ & $2.21 \pm 0.12$ & $1.98 \pm 0.08$ & 0.07 & 0.67 & 0.13 & 0.77 \\
\hline Butyrate, C4 & $2.28^{\mathrm{b}} \pm 0.17$ & $1.93^{\mathrm{ab}} \pm 0.09$ & $1.65^{\mathrm{a}} \pm 0.17$ & $2.07 \pm 0.13$ & $1.84 \pm 0.13$ & 0.09 & $0.02 *$ & 0.20 & 0.95 \\
\hline TVFA & $13.65 \pm 0.47$ & $13.36 \pm 0.62$ & $12.39 \pm 0.50$ & $13.76^{p} \pm 0.42$ & $12.50^{\circ} \pm 0.43$ & 0.31 & 0.22 & $* 0.045$ & 0.64 \\
\hline Acetate, C2 & $65.77^{a} \pm 0.68$ & $67.64^{\mathrm{ab}} \pm 0.50$ & $69.26^{\mathrm{b}} \pm 1.15$ & $67.49 \pm 0.64$ & $67.62 \pm 0.84$ & 0.52 & $0.02 *$ & 0.44 & 0.45 \\
\hline Propionate, C3 & $15.77 \pm 0.53$ & $15.86 \pm 0.46$ & $16.11 \pm 0.55$ & $16.02 \pm 0.53$ & $15.81 \pm 0.26$ & 0.29 & 0.29 & 0.24 & 0.60 \\
\hline Butyrate, C4 & $16.58^{\mathrm{b}} \pm 0.89$ & $14.49^{\mathrm{ab}} \pm 0.41$ & $13.14^{\mathrm{a}} \pm 1.31$ & $14.99 \pm 0.77$ & $14.48 \pm 0.89$ & 0.58 & $0.02 *$ & 0.44 & 0.62 \\
\hline$A: P$ or $(C 2: C 3$ ratio $)$ & $4.22 \pm 0.13$ & $4.30 \pm 0.13$ & $4.34 \pm 0.13$ & $4.28 \pm 0.13$ & $4.29 \pm 0.07$ & 0.07 & 0.32 & 0.30 & 0.48 \\
\hline $\begin{array}{l}(A+B): P \\
\text { or }(C 2+C 4): C 3 \text { ratio }\end{array}$ & $5.29 \pm 0.20$ & $5.23 \pm 0.17$ & $5.18 \pm 0.19$ & $5.25 \pm 0.19$ & $5.21 \pm 0.09$ & 0.10 & 0.33 & 0.21 & 0.47 \\
\hline
\end{tabular}

Mean bearing different superscripts in a column and row differ significantly, $* p<0.05$; TVFA=Total volatile

fatty acids, $\mathrm{mM} / 100 \mathrm{ml}=$ Milli-mole per 100 millilitre. C2:C3 ratio=Acetate:propionate ratio, (C2+C4):C3

ratio $=($ Acetate+butyrate $):$ Propionate ratio. TMR=Total mixed ration, $\mathrm{SEM}=$ Standard error of mean, $\mathrm{SE}=\mathrm{Standard}$ error, $\mathrm{T}=$ Dietary treatment, $\mathrm{S}=$ Species (crossbred cattle and buffalo), $\mathrm{T} \times \mathrm{S}=$ Interaction between species and dietary treatments, TVFA=Total volatile fatty acids, $\mathrm{C}=$ Concentrate mixture, $\mathrm{W}=$ Wheat straw, $\mathrm{G}=\mathrm{Green}$ maize

concentrations were found to be $2.28 \pm 0.17,1.93 \pm 0.09$, and $1.65 \pm 0.17$ for treatments $\mathrm{T}_{1}, \mathrm{~T}_{2}$, and $\mathrm{T}_{3}$, respectively. The difference was significant $(p<0.05)$ between $T_{1}$ and $T_{3}$ group while $T_{2}$ differed non-significantly $(\mathrm{p}>0.05)$ from both of them.

The molar percent of acetate, propionate, butyrate, $A: P$ ratio and $(A+B): P$ ratio varied non-significantly $(p>0.05)$ between crossbred cattle and buffaloes. Although molar proportion of acetate and butyrate $(\mathrm{mol} / 100 \mathrm{~mol})$ differed significantly $(\mathrm{p}<0.05)$ among different treatment groups. Acetate proportion was found to be highest in green fodder rich diet $\left(\mathrm{T}_{3}\right)$ while butyrate proportion was found to be highest in concentrate fodder rich diet $\left(\mathrm{T}_{1}\right)$ (Table-5).

\section{Rumen enzyme activities}

Rumen liquor was collected from all animals and analyzed for rumen enzyme, viz., CMCase, xylanase, avicelase, and protease (Table-6). The result indicated that the microbial enzyme profiles of crossbred cattle and buffaloes were comparable ( $p>0.05$ ). No significant $(p>0.05)$ variation in rumen microbial enzyme activity was also observed among the treatment group animals post-feeding.

\section{Ciliate protozoa}

Among the treatment groups, protozoal population of holotrichous and sporotrichosis were found to be significantly higher $(\mathrm{p}<0.01)$ in $\mathrm{T}_{1}$ and $\mathrm{T}_{2}$ than $\mathrm{T}_{3}$ group animals, whereas cross-bred cattle population was found to harbor significantly $(p<0.01)$ higher number of both of the protozoa than buffaloes (Table-7). Total protozoa population was significantly $(p<0.01)$ higher in crossbred cattle than buffaloes, but within treatment groups, it was highest for $\mathrm{T}_{1}$ and lowest for $\mathrm{T}_{3}$ group animals.

\section{Discussion}

Feeding of different concentrate roughage ratios was found to have no drastic effect on rumen environment [29]. In crossbred cattle and buffalo, mean value of rumen $\mathrm{pH}$ was in agreement with values reported by Franzolin et al. [16] and Baraka [30]. A similar type of non-significant $(\mathrm{p}>0.05)$ effect on diurnal ruminal $\mathrm{pH}$ was also documented by Chanthakhoun et al. [1] between swamp buffalo and beef cattle fed on rice straw.

There was no significant variation in protease activity between buffalo and cattle, but from the 
Table-6: Effect on enzyme activities (IU/mg protein) in rumen content of fistulated crossbred cattle and buffaloes fed various TMR diet.

\begin{tabular}{|c|c|c|c|c|c|c|c|c|c|}
\hline \multirow{2}{*}{$\begin{array}{l}\text { Enzyme } \\
\text { activity }\end{array}$} & \multirow{2}{*}{$\begin{array}{c}\text { 60C: } 20 \mathrm{~W}: \\
\text { 20G } \\
\text { T1 }\end{array}$} & \multirow{2}{*}{$\begin{array}{c}\text { 40C:30W: } \\
\text { 30G } \\
\text { T2 }\end{array}$} & \multirow{2}{*}{$\begin{array}{c}\text { 20C:40W: } \\
\text { 40G } \\
\text { T3 }\end{array}$} & \multicolumn{2}{|c|}{ Mean \pm SE } & \multirow[t]{2}{*}{ SEM } & \multicolumn{3}{|c|}{ p values } \\
\hline & & & & Cattle & Buffalo & & $\mathbf{T}$ & $\mathbf{S}$ & $\mathbf{T} \times \mathbf{S}$ \\
\hline & $6.37 \pm 3.81$ & $60.78 \pm 3.89$ & $6000+316$ & $30+2$ & 27012 & & 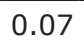 & .76 & 0 . \\
\hline Xylanase & $179.55 \pm 15.18$ & $200.51 \pm 15.85$ & $222.33 \pm 11.05$ & $213.43 \pm 13.12$ & $188.16 \pm 10.27$ & 8.48 & 0.12 & 0.13 & 0.70 \\
\hline Avicelase & $27.35 \pm 2.93$ & $29.20 \pm 2.16$ & $31.79 \pm 1.84$ & $31.35 \pm 2.31$ & $27.55 \pm 1.33$ & 1.35 & 0.41 & 0.17 & 0.55 \\
\hline Protease & $162.92 \pm 8.33$ & $165.69 \pm 7.00$ & $157.66 \pm 8.01$ & $158.82 \pm 7.28$ & $165.36 \pm 5.07$ & 4.41 & 0.77 & 0.49 & 0.97 \\
\hline
\end{tabular}

Unit=nmol of glucose released $/ \mathrm{min} / \mathrm{ml}$ for CMCase and avicelase; $\mathrm{nmol}$ of $\mathrm{xylose}$ released $/ \mathrm{min} / \mathrm{ml}$ for $\mathrm{xylanase}$;

$\mu \mathrm{g}$ hydrolyzed protein/min/ml for protease; The mean values did not differ significantly at level $p<0.05$.

$\mathrm{IU} / \mathrm{mg}=$ International unit per milligram, CMCase=Carboxymethylcellulase, TMR=Total mixed ration, SEM=Standard error of mean, $\mathrm{SE}=$ Standard error, $\mathrm{T}=$ Dietary treatment, $\mathrm{S}=\mathrm{Species}$ (crossbred cattle and buffalo), $\mathrm{T} \times \mathrm{S}=$ Interaction between species and dietary treatments, $\mathrm{C}=$ Concentrate mixture, $\mathrm{W}=$ Wheat straw, $\mathrm{G}=\mathrm{Green}$ maize

Table-7: Effect on protozoal population (Log10) of fistulated crossbred cattle and buffaloes fed various TMR diet.

\begin{tabular}{|c|c|c|c|c|c|c|c|c|c|}
\hline \multirow{2}{*}{$\begin{array}{l}\text { Rumen } \\
\text { protozoa }\end{array}$} & \multirow{2}{*}{$\begin{array}{c}\text { 60C: 20W: } \\
\text { 20G } \\
\text { T1 }\end{array}$} & \multirow{2}{*}{$\begin{array}{c}\text { 40C: } 30 W: \\
30 G \\
T 2\end{array}$} & \multirow{2}{*}{$\begin{array}{c}\text { 20C: 40W: } \\
\text { 40G } \\
\text { T3 }\end{array}$} & \multicolumn{2}{|c|}{ Mean \pm SE } & \multirow[t]{2}{*}{ SEM } & \multicolumn{3}{|c|}{ p values } \\
\hline & & & & Cattle & Buffalo & & $\mathbf{T}$ & $\mathbf{S}$ & $\mathbf{T} \times \mathbf{S}$ \\
\hline & & & & D & 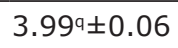 & & . & . & \\
\hline Spirotrichous & $5.36^{b} \pm 0.04$ & $5.27^{\mathrm{a}} \pm 0.05$ & $5.23^{a} \pm 0.0$ & $5.43^{p} \pm 0.02$ & & & $0.008 * *$ & $0.009 * *$ & 0.7 \\
\hline Total protozoa & $5.40^{\complement} \pm 0.04$ & $5.31^{\mathrm{b}} \pm 0.05$ & $5.26^{\mathrm{a}} \pm 0.04$ & $5.46^{p} \pm 0.02$ & $5.18^{a} \pm 0.02$ & 0.03 & $0.008 * *$ & $0.008 * *$ & 0.85 \\
\hline
\end{tabular}

Mean bearing different superscripts in a column and row differ significantly. ${ }^{*} p<0.05$; Log=Logarithm, TMR=Total mixed ration, $\mathrm{SEM}=$ Standard error of mean, $\mathrm{SE}=$ Standard error, $\mathrm{T}=$ Dietary treatment, $\mathrm{S}=\mathrm{Species}$ (crossbred cattle and buffalo), $\mathrm{T} \times \mathrm{S}=$ Interaction between species and dietary treatments, $\mathrm{C}=$ Concentrate mixture, $\mathrm{W}=\mathrm{Wheat}$ straw, $\mathrm{G}=\mathrm{Green}$ maize

enzyme table, it is evident that protease activity is higher in buffaloes $(165.36 \pm 5.07 \mathrm{IU} / \mathrm{mg}$ protein) than cattle $(158.82 \pm 7.28 \mathrm{IU} / \mathrm{mg}$ protein). The difference may not be significant but its effect may be enough leading to higher NH3-N concentration in buffaloes. Ruminal $\mathrm{NH}_{3}-\mathrm{N}$ varied significantly $(\mathrm{p}<0.05)$ and was found to be higher in buffalo than crossbred cattle which was in agreement with Khajarern and Khajarern [31]. The higher concentration of $\mathrm{NH}_{3}-\mathrm{N}$ in buffaloes indicates higher proteolytic activity in the rumen of buffaloes than crossbred cattle although the variation in enzyme activity was not significant [32]. Suwanlee and Wanapat [33] reported that when ruminal $\mathrm{NH}_{3}-\mathrm{N}$ increased from 1.7 to $5.6 \mathrm{mg} \%$, total bacterial count, digestibility of DM, NDF and ADF were increased. In the current study, it is evident from Tables-3 and 4 that the overall trend suggests an increase in ruminal NH3-N level occurs along with increase in DM intake (DMI) among different feeding groups, although the level of variation in terms of significance was not found exactly similar. In case of interspecies difference, buffaloes have higher ruminal NH3-N than cattle along with higher DMI, although the difference in DMI was non-significant. We have removed protozoal population from this context in the revised manuscript as protozoal population is largely dependent on species of animal, type, and source of diet which we have mentioned in the later part of the manuscript. Increased level of ruminal $\mathrm{NH}_{3}-\mathrm{N}$ resulted from increased DMI [29]. However, Chanthakhoun et al. [1] reported that NH3-N concentration of buffalo did not differ from cattle although nutrient digestibilities particularly those of DM, OM, $\mathrm{CP}, \mathrm{NDF}$, and ADF was significantly $(\mathrm{p}<0.05)$ higher in buffalo than cattle.

The values of TN, TCA ppt. N (mg \%) and LA $(\mathrm{mg} / \mathrm{ml})$ in the rumen liquor increased significantly $(p<0.05)$ with the increase in the proportion of concentrate mixture in the diet of animals. This could be due to increased soluble carbohydrates and proteins in high concentrate diet. The increased nitrogen fractions might be due to increase in the nitrogen intake by the animals with increasing proportion of concentrate in the diet. Higher concentration of ammonia reflecting better activity of intracellular deaminases and salivary recycling of urea [34] and maintenance of nitrogen balance positive due to the greater efficiency of utilization of $\mathrm{NH}_{3} \mathrm{~N}$ by ruminal bacteria [35].

Wanapat and Pimpa [29] reported that LA $(\mathrm{mg} / \mathrm{ml})$ in the rumen liquor increased significantly $(p<0.05)$ with the increase in the proportion of concentrate mixture in the diet of animals; however, in the present study, there was no such effect observed on LA concentration between and within the treatment groups.

Significantly $(p<0.05)$ higher TVFAs concentration was observed in the rumen liquor of crossbred cattle than buffaloes in the current study which was in similar trend with Chanthakhoun et al. [1] and Franzolin et al. [16] where higher VFA concentration was observed in cattle than buffaloes although the difference was non-significant, while Cutrignelli et al. [36] reported that buffaloes produced higher rumen VFAs than cattle. Although several factors 
such as anatomy and physiology of digestive system, feed intake and digestibility, rumen microbial metabolism, and rumen ciliate protozoal population $[37,38]$ may be involved for this interspecies variation in VFA production but the exact metabolic mechanism is not known [39]. However, it has been observed that the $\mathrm{pH}$ and ammonia concentration of rumen liquor decreased while production of TVFA increased in the absence of rumen ciliate protozoa in lambs [40]. TVFAs were found at normal concentrations of $70-130 \mathrm{mmol} / \mathrm{L}$ and also proportions of acetate, propionate, and butyrate in this study were in accordance with Hungate [41]. Among treatments, variation in the level of TVFA was non-significant $(p>0.05)$. The proportion of VFAs (acetate, propionate, and butyrate) was not affected by energy sources which were in agreement with Hoover et al. [42].

Franzolin et al. [16] described buffaloes had lower production of acetic acid than cattle (58.7 vs. $61.6 \mathrm{~mol} / 100 \mathrm{~mol}$ ) and higher proportion of propionic acid ( 27.4 vs. $23.6 \mathrm{~mol} / 100 \mathrm{~mol})$. There was no difference in the butyric acid production between the buffaloes $(13.6 \mathrm{~mol} / 100 \mathrm{~mol})$ and cattle $(14.8 \mathrm{~mol} / 100 \mathrm{~mol})$.

Enzyme activities in terms of CMCase, xylanase, avicelase, and protease activity were found to be similar in rumen content of cattle and buffalo. The probable reason of similar enzyme activity in these two species could be due to the same microbial population in the rumen fed with similar type of diets. Some workers reported higher cellulose digestibility in buffaloes than cattle [43], whereas others did not observe any difference [44].

Total protozoa population was significantly $(p<0.01)$ higher in crossbred cattle than buffaloes but within treatment groups and it was highest for concentrate rich diet $\left(\mathrm{T}_{1}\right)$ and lowest for low concentrate diet $\left(\mathrm{T}_{3}\right)$ group animals. It was in agreement with Franzolin et al. [16] who reported similar type of interspecies difference in total rumen protozoal count. They also reported that protozoal count and type may vary according to the type and source of diet. Other previous reports also depicted that zebu cattle had higher numbers of rumen protozoa than the buffaloes $[1,45]$; however inversely, it was higher in buffalo than in cattle rumen [3], but no differences were reported by Kurar et al. [46].

\section{Conclusion}

From the present study, it may be concluded that optimum roughage to concentrate is one of the dietary means which influence microbial population for rumen fermentation depending on constituents of the treatment diet. Activities of microbial enzymes were comparable among species and different treatment groups. A total number of rumen protozoa were higher in crossbred cattle than buffaloes along with higher in animal fed high concentrate diet. Further, comparison of the rumen metabolism in crossbred cattle and buffaloes could be of scientific interest to improve the rumen microbial activities of these species by manipulating ruminal microenvironment for better digestibility and utilization of the similar feedstuffs.

\section{Authors' Contributions}

SKS processed and analyzed the samples. VBC, PS, and LCC designed the study and corrected the manuscript. SKS, MG, and SS analyzed the data and prepared the manuscript. All authors read and approved the final manuscript.

\section{Acknowledgments}

The authors wish to express their gratitude to the Rumen Microbiology Lab, Animal Nutrition Division and the Director, ICAR-IVRI, Izatnagar, Bareilly, Uttar Pradesh, India, for providing financial support for the current study.

\section{Competing Interests}

The authors declare that they have no competing interests.

\section{References}

1. Chanthakhoun, V., Wanapat, M., Kongmun, P. and Cherdthong, A. (2012) Comparison of ruminal fermentation characteristics and microbial population in swamp buffalo and cattle. Livest. Sci., 143: 172-176.

2. Lwin, K.O., Kondo, M., Ban-Tokuda, T., Lapitan, R.M., Del-Barrio, A.N., Fujihara, T. and Matsui, H. (2012) Ruminal fermentation and microbial ecology of buffaloes and cattle fed the same diet. Anim. Sci. J., 83: 767-776.

3. Jabari, S., Eslami, M., Chaji, M., Mohammadabadi, T. and Bojarpour, M. (2014) Comparison digestibility and protozoa population of Khuzestan water buffalo and Holstein cow. Vet. Res. Forum, 5(4): 295-300.

4. Franzolin, R. and Wright, A.G. (2016) Microorganisms in the rumen and reticulum of buffalo (Bubalus bubalis) fed two different feeding systems. BMC Res. Notes, 9: 243.

5. Khejornsart, P., Wanapat, M. and Rowlinson, P. (2011) Diversity of anaerobic fungi and rumen fermentation characteristic in swamp buffalo and beef cattle fed on different diets. Livest. Sci., 139(3): 230-236.

6. Rafiei, M., Chaji, M., Mohammadabadi, T. and Sari, S. (2013) The comparison digestibility of steam treated sugarcane pith by rumen bacteria or rumen microorganisms of Holstein cow and buffalo of Khuzestan. J. Rumin. Res., 1(1): 53-75.

7. Vinh, N.T., Wanapat, M., Khejornsart, P. and Kongmun, P. (2011) Studies of diversity of rumen microorganisms and fermentation in swamp buffalo fed different diets. J. Anim. Vet. Adv., 10: 406-414

8. Wright, A.D.G. and Klieve, A.V. (2011) Does the complexity of the rumen microbial ecology preclude methane mitigation? Anim. Feed. Sci. Technol., 166: 248-253.

9. Chaji, M. and Mohammadabadi, T. (2011) The investigation of in vitro fermentation of sugarcane pith treated with low temperature steam and sulfuric acid by isolated rumen microbial fractions. Anim. Nutr. Feed. Technol., 11: 185-193.

10. Franzolin, R., St-Pierre, B., Northwood, K. and Wright, A.D.G. (2012) Analysis of rumen methanogen diversity in water buffaloes (Bubalus bubalis) under three different diets. Microb. Ecol., 64: 131-139.

11. Wanapat, M., Pilajun, R., Polyorach, S., Cherdthong, A., Khejornsart, P. and Rowlinson, P. (2013) Effect of carbohydrate source and cottonseed meal level in the concentrate on feed intake, nutrient digestibility, rumen fermentation and 
microbial protein synthesis in swamp buffaloes. Asian Aust. J. Anim. Sci., 26: 952-960.

12. Nathani, N.M., Patel, A.K., Dhamannapatil, P.S., Kothari, R.K., Singh, K.M. and Joshi, C.G. (2013) Comparative evaluation of rumen metagenome community using qPCR and MG-RAST. AMB Expr., 3: 55.

13. Shakarami, F., Chaji, M., Eslami, M., Mohammadabadi, T. and Bojarpour, M. (2015) The comparison of in vitro digestibility of wheat straw by rumen anaerobic fungi of Khuzestan buffalo and Holstein cattle. Iran J Apppl. Anim. Sci., 5(2): 285-292.

14. Wadhwa, M., Bakshi, M.P.S. and Makkar, H.P.S. (2016) Modifying gut microbiomes in large ruminants: Opportunities in non-intensive husbandry systems. Anim. Front., 6(2): 27-36.

15. Wanapat, M., Sommart, K., Wachirapakorn, C., Uriyapongson, S. and Wattanachant, C. (1994) Recent advances in swamp buffalo nutrition and feeding. In: Wanapat, M. and Sommart, K., editors. Proceeding of the $1^{\text {st }}$ Asian Buffalo Association Congress. January 17-21. Khon Kaen University, Khon Kaen, Thailand.

16. Franzolin, R., Rosales, F.P. and Soares, W.V.B. (2010) Effects of dietary energy and nitrogen supplements on rumen fermentation and protozoa population in buffalo and zebu cattle. Rev. Bras. Zootec., 39: 549-555.

17. Wanapat, M., Phesatcha, K. and Kang, S. (2016) Rumen adaptation of swamp buffaloes (Bubalus bubalis) by high level of urea supplementation when fed on rice straw-based diet. Trop. Anim. Health Prod., 48(6): 1135-1140.

18. van Soest, P.J. (1994) Nutritional Ecology of the Ruminant. $2^{\text {nd }}$ ed. Cornell University Press, Ithaca, NY.

19. Weatherburn, M.W. (1967) Phenol hypochlorite reaction for determination of ammonia. Anal. Chem., 39: 971-974.

20. Cottyn, B.G. and Boucque, C.V. (1968) Rapid method for the gas chromatographic determination of volatile fatty acids in rumen fluid. J. Agric. Food Chem., 16: 105-107.

21. AOAC. (1995) Association of Official Analytical Chemist. $16^{\text {th }}$ ed. Association of Official Analytical Chemists, Washington, DC.

22. Barker, S.B. and Summerson, W.H. (1941) The calorimetric determination of lactic acid in biological materials. J. Biol. Chem., 138: 535-554

23. Hristov, A.N., McAllister, T.A. and Cheng, K.J. (1999) Effect of diet, digesta processing, freezing and extraction procedure on some polysaccharide degrading activities of ruminal contents. Can. J. Anim. Sci., 79: 73-81.

24. Miller, G.L. (1959) Modified DNS method for reducing sugars. Anal. Chem., 31: 426-428.

25. Lowry, O.H., Rosenbrough, N.J., Farr, A.L. and Randall, R.C. (1951) Protein measurement with the folin-phenol reagent. J. Biol. Chem., 183: 265.

26. Brock, F.M., Forsberg, C.L. and Buchanan-Smith, J.G. (1982) Proteolytic activity of rumen microorganisms and effects of proteinase inhibitors. Appl. Environ. Microbiol., 44: 561-569.

27. Kamra, D.N., Sawal, R.K., Pathak, N.N., Kewalramani, N. and Agarwal, N. (1991) Diurnal variation in ciliate protozoa in the rumen of black buck (Antilope cervicapra) fed green forage. J. Appl. Microbiol., 13: 165-167.

28. Snedecor, G.W. and Cochran, W.G. (1994) Statistical Methods. $8^{\text {th }}$ ed. Iowa State University Press, Ames.

29. Wanapat, M. and Pimpa, O. (1999) Effect of ruminal $\mathrm{NH}_{3}-\mathrm{N}$ levels on ruminal fermentation, purine derivatives, digestibility and rice straw intake in swamp buffaloes. Asian Australas. J. Anim. Sci., 12: 904-907.

30. Baraka, T.A. (2012) Comparative studies of rumen $\mathrm{pH}$, total protozoa count, generic and species composition of ciliates in camel, buffalo, cattle, sheep and goat in Egypt. J. Am.
Sci., 8(2): 655-669.

31. Khajarern, S. and Khajarern, J.M. (1990) Feeding swamp buffalo for milk production. Feeding dairy cows in the tropics. FAO Anim. Prod. Health Pap., 86: 115-126.

32. Bhatia, S.K., Pradhan, K., Sangwan, D.C., Singh, S. and Sagar, V. (1995) Ruminal degradation of fibrous component of various feeds in cattle and buffalo. Indian J. Anim. Sci., 65: 208.

33. Suwanlee, S. and Wanapat, M. (1994) Effect of ruminal $\mathrm{NH}_{3}-\mathrm{N}$ on total volatile fatty acid, bacterial population and digestibility in swamp buffaloes. In: Wanapat, M. and Sommart, K., editors. Proceedings of the $1^{\text {st }}$ Asian Buffalo Association Congress. Khon Kaen University, Thailand.

34. Tewatia, B.S. and Bhatia, S.K. (1998) Comparative ruminal biochemical and digestion related physiological characteristics in buffaloes and cattle fed a fibrous diet. Buffalo J., 14: $161-170$.

35. Gandra, J.R., Freitas, J.E.Jr., Barletta, R.V., Filho, M.M., Gimenes, L.U., Vilela, F.G., Baruselli, P.S. and Rennó, F.P. (2011) Productive performance, nutrient digestion and metabolism of Holstein (Bos taurus) and Nellore (Bos taurus indicus) cattle and Mediterranean Buffaloes (Bubalis bubalis) fed with corn-silage based diets. Livest. Sci., 140: 283-291.

36. Cutrignelli, M.I., D'urso, S., Tudisco, R., Grossi, M. and Piccolo, V. (2007) Effect of ruminant species (Bovine vs buffalo) and source of inoculum (rumen liquor vs faeces) on in vitro fermentation. Ital. J. Anim. Sci., 6: 295-297.

37. Franzolin, R. (1994) Feed efficiency: A comparison between cattle and buffalo. Buffalo J. Suppl., 2: 39-50.

38. Calabro, S., Moniello, G., Piccolo, V., Bovera, F., Infascelli, F., Tudisco, R. and Cutrignelli, M.I. (2008) Rumen fermentation and degradability in buffalo and cattle using the in vitro gas production technique. J. Anim. Physiol. Anim. Nutr., 92: 356-362.

39. Calabrò, S., Williams, B.A., Piccolo, V., Infascelli, F. and Tamminga, S. (2004) A comparison between buffalo (Bubalus bubalis) and cow (Bos taurus) rumen fluids in terms of the in vitro fermentation characteristics of three fibrous feedstuffs. J. Sci. Food Agric., 84: 645-652.

40. Santra, A. and Karim, S.A. (2002) Influence of ciliate protozoa on biochemical changes and hydrolytic enzyme profile in the rumen ecosystem. J. Appl. Microbiol., 92: 801-811.

41. Hungate, R.E. (1966) The Rumen and its Microbes. Academic Press, New York. p533.

42. Hoover, W.H., Tucker, C., Harris, J., Sniffen, C.J. and de Ondarza, M.B. (2006) Effects of non-structural carbohydrate level and starch: Sugar ratio on microbial metabolism in continuous culture of rumen contents. Anim. Feed. Sci. Technol., 128: 307-319.

43. Ichinohe, T., Orden, E.A., Delbarrio, A.N., Lapitan, R.M., Fujihara, T., Cruz, L.C. and Kanai, Y. (2004) Comparison of voluntary feed intake, rumen passage and degradation kinetics between crossbred Brahmam cattle (Bos indicus) and swamp buffaloes (Bubalus bubalis) fed a fattening diet based on corn silage. J. Anim. Sci., 75: 533-540.

44. Lapitan, R.M., Del-Barrio, A.N., Katsube, O., Ban-Tokuda, T., Orden, E.A., Robles, A.Y., Kanai, L.C., Cruz, Y. and Fujihara, T. (2008) Comparison of fattening performance in Brahman grade cattle (Bos indicus) and crossbred water buffalo (Bubalus bubalis) fed on high roughage diet. J. Anim. Sci., 79: 76-82.

45. Dehority, B.A. (2003) Rumen Microbiology. Nottingham University Press, Thrumpton, p372.

46. Kurar, C.K., Gupta, B.N. and Mohini, M. (1988) Protozoal status in strained rumen liquor of cattle and buffaloes. Indian J. Anim. Sci., 58: 112-115. 